\title{
Mood Alteration
}

National Cancer Institute

\section{Source}

National Cancer Institute. Mood Alteration. NCI Thesaurus. Code C62592.

A change in disposition or state of mind. 